CHAPITRE 1

\title{
La filière Tokamak et la machine Iter
}

ROBERT AYMAR ET GUY LAVAL 

Pour pouvoir produire de l'énergie à partir des réactions de fusion entre des noyaux de deutérium et des noyaux de tritium, la température du mélange doit dépasser cent millions de degrés et la densité des particules doit être suffisamment élevée $\left(\mathrm{n} \approx 10^{20} \mathrm{p} / \mathrm{m}^{3}\right)$. Pour réaliser ces conditions physiques de manière contrôlée et permanente, il reste à trouver une solution assurant une isolation thermique suffisante du gaz chaud pour que les particules ne perdent pas trop d'énergie avant de réagir. Le confinement magnétique des plasmas donne une solution à ce problème inhabituel. II utilise la forte interaction qui se développe entre le gaz et un champ magnétique à ces énormes températures. En effet, dans de telles conditions, les électrons sont arrachés des noyaux par les collisions entre les particules, et le gaz ne contient plus que des particules chargées libres. Les électrons et les noyaux portent des charges de signes opposés de telle sorte que le milieu reste neutre en moyenne : le gaz est devenu un plasma, par définition. Un champ magnétique ou un champ électrique se couplent à ces particules chargées en exerçant des forces sur ce plasma.

La liberté retrouvée des électrons permet au plasma de conduire facilement l'électricité et donc de porter un courant. Plongé dans un champ magnétique, le plasma pourra alors subir une force distribuée dans tout son volume et capable d'équilibrer la pression du gaz ionisé. C'est le principe du confinement magnétique des plasmas. L'idée la plus simple consiste à imaginer un plasma en forme d'anneau et parcouru par un courant, entouré par une coque conductrice de même forme. Malheureusement, la configuration est instable, le plasma est soumis à des mouvements désordonnés et son confinement est détruit. Pour la stabiliser, il faut ajouter un champ magnétique supplémentaire, généré par une série de bobines régulièrement réparties le long de l'anneau de plasma. La stabilité demande que ce champ additionnel domine en intensité celvi qui est généré par le courant du plasma. Quelques bobines coaxiales avec l'anneau permettent de changer la forme de sa section en contrôlant la limite définissant le plasma chaud, à une certaine distance des parois de l'enceinte. La configuration Tokamak part de ces principes simples.

Pour porter le plasma ainsi piégé à la température convenant à la fusion, des sources de rayonnement ou de particules injectent de l'énergie qui se transforme en chaleur dans le milieu. Pour que l'équilibre obtenu soit compatible avec la forme et les dimensions de l'enceinte, les champs des bobinages et celui créé par le courant du plasma doivent remplir des conditions qui se traduisent grossièrement par une valeur déterminée du rapport entre ces deux composantes au bord du plasma. Une configuration magnétique $n^{\prime}$ assure pas le confinement $d^{\prime}$ un plasma de $n^{\prime}$ importe quelle pression. Elle impose une limite supérieure au rapport entre la pression cinétique du plasma et la densité d'énergie magnétique et cette limite dépend du courant dans le plasma et de la géométrie choisie. Toutes ces contraintes réduisent considérablement la liberté de choix pour les paramètres définissant un Tokamak. Ainsi s'expliquent les analogies 
de comportement observées dans les différentes machines de tailles diverses et utilisant les mêmes concepts, construites dans le monde entier au fil des années.

Malheureusement, les performances d'un Tokamak ne peuvent pas se déterminer seulement à partir de ces considérations simples. Elles permettent seulement d'assurer l'existence d'un équilibre macroscopique et d'éviter les instabilités les plus destructrices. II n'existe pas de moyen, aujourd'hui, permettant de stabiliser le plasma à toutes les échelles de temps et de longueur. Ces instabilités résiduelles maintiennent l'existence de champs électromagnétiques turbulents, avec des fluctuations dont l'amplitude reste assez faible pour être tolérable, mais qui déterminent le transport des particules à travers la configuration magnétique et, donc, la vitesse à laquelle le plasma s'échappe du volume de confinement.

Cette vitesse conditionne le bilan énergétique de la configuration et sa détermination constitue le premier objectif poursuivi dans les recherches sur la fusion par confinement magnétique. Devant l'impossibilité d'un calcul a priori, la similarité des régimes de fonctionnement dans les différentes machines a permis d'établir des lois empiriques en rassemblant l'ensemble des résultats. Leur extrapolation guide la conception des machines nouvelles et permet des prédictions sur leurs performances mais elles n'autorisent que des sauts d'amplitude relativement faibles et ne peuvent prédire les changements de régimes de fonctionnement. Par exemple, les premières lois avaient été établies pour des puissances de chauffage faibles et $n^{\prime}$ ont pas permis de prévoir un important changement de régime au-delà d'un seuil qui s'est manifesté en effectuant des études systématiques sur un Tokamak de taille moyenne, la machine Asdex. Plus récemment, les expériences sur plusieurs machines ont mis en évidence d'autres régimes de confinement amélioré en effectuant un contrôle fin de la distribution du courant dans le plasma. Là encore, l'absence d'un modèle entièrement calculable rend difficile l'évaluation des gains espérés de tels effets. Ces limites à l'extrapolation justifient la construction d'une expérience où il soit possible de juger de la valeur réelle de ces progrès dans des conditions jugées directement transposables à un réacteur. C'est la motivation principale qui a conduit à la décision de construire la machine Iter.

\section{1 | La machine lter}

La configuration Tokamak se définit par le grand rayon $\mathrm{R}$ et le petit rayon a du tore de plasma, le courant électrique I circulant dans le plasma, la valeur du champ magnétique additionnel stabilisateur B. Il faut y ajouter la densité $\mathrm{n}$ et la température $\mathrm{T}$ du plasma ainsi que les données géométriques définissant la forme du tore, lorsqu'il n'est pas à section circulaire. Ainsi, pour lter, la 
configuration nominale correspond à : $R=6,2 \mathrm{~m}, \mathrm{a}=2,0 \mathrm{~m}, \mathrm{I}=15$ à 17 millions $d$ 'ampères, $B=5,3$ Teslas, forme allongée avec une élongation verticale de 1,84. La température du plasma avoisine les $20 \mathrm{keV}$ (200 millions de degrés environ) mais la densité ne dépasse pas $10^{20}$ particules par $\mathrm{m}^{3}$, soit un cent millième de la densité de l'air à la pression atmosphérique. La pression reste modérée, de l'ordre de quelques atmosphères.

Dix-huit bobines supraconductrices génèrent le champ magnétique de stabilisation dans une enceinte à vide torique où les $837 \mathrm{~m}^{3}$ de plasma restent en équilibre, éloignés des parois, et sont chauffés par différentes sources d'énergie qui injectent $73 \mathrm{MW}$ sous forme de particules ou de rayonnement électromagnétique. D'autres circuits créent les champs magnétiques auxiliaires nécessaires pour donner à la section du plasma la forme désirée. L'ensemble du Tokamak est enfermé à l'intérieur d'un cryostat contenant des écrans, refroidis à $80 \mathrm{~K}$, entre les éléments chauds et les aimants supraconducteurs maintenus à $4,5 \mathrm{~K}$. La machine tient dans un cylindre de $28 \mathrm{~m}$ de diamètre et $24 \mathrm{~m}$ de hauteur.

Le plasma restera isolé des parois grâce à une installation, appelée divertor. Elle assure l'évacuation vers l'extérieur des ions et des électrons qui franchissent une surface torique déterminée, définissant le bord du plasma. Le profil du champ magnétique assure le guidage de ces particules vers une chambre, située dans la partie inférieure de la machine, où elles subissent un refroidissement radiatif avant une collecte sur des plaques métalliques qui les neutralisent et permettent de les évacuer par pompage. Le divertor concentre ainsi le contact direct entre les parois et le plasma chaud assez loin du bord du plasma, au prix $d^{\prime}$ une contrainte thermique très forte sur les plaques. Le refroidissement radiatif préalable du plasma permettra de limiter le flux de chaleur sur les plaques audessous de la limite supportable de $10 \mathrm{MW} / \mathrm{m}^{2}$. La mise au point de ce divertor conditionnera l'évolution de ces configurations vers de futurs réacteurs et fait partie des enjeux majeurs d'lter.

\section{2 | Objectif nominal}

Le déroulement d'une expérience peut suivre plusieurs scénarios selon l'objectif poursuivi. La validation du Tokamak comme voie vers le réacteur fixe le but final d'Iter : optimiser la configuration de l'étape suivante sur le chemin menant à l'exploitation de la fusion comme source d'énergie industrielle. Mais la recherche des conditions qui s'en rapprochent le mieux s'effectuera avec une certaine progressivité. Pendant les phases de mise au point, Iter fonctionnera avec de l'hydrogène, puis avec du deutérium. Dans ce dernier cas, les quelques neutrons produits serviront de diagnostic. Une fois la machine bien prise en 
main, l'effort se concentrera sur le premier objectif proposé par l'équipe de conception et accepté par les pays partenaires:

« Le dispositif devra réaliser la combustion dans un plasma généré par induction avec une puissance produite au moins dix fois supérieure à la puissance du chauffage auxiliaire, dans des domaines de fonctionnement et avec une durée suffisante pour réaliser des conditions stationnaires par rapport aux temps caractéristiques des processus gouvernant la physique du plasma. »

L'induction se réfère à la méthode utilisée pour générer le courant dans le plasma torique. La technique correspondante imite le principe des transformateurs électriques : le courant du plasma est maintenu par la force électromotrice obtenue en faisant varier dans le temps le flux magnétique embrassé par l'anneau de plasma. Cette méthode simple présente l'inconvénient de ne fonctionner que sur une durée finie et ne peut convenir à un système stationnaire. Mais la condition imposée se limite à obtenir la stationnarité par rapport à des temps caractéristiques qui vont de quelques secondes à quelques minutes. Dans ces conditions, l'induction convient puisqu'elle est compatible avec des décharges de 300 à 400 secondes, qui remplissent la condition imposée.

En revanche, la performance demandée pour la puissance produite implique un plasma de deutérium et de tritium, produisant d'importantes émissions de neutrons et de noyaux d'hélium énergétiques (respectivement $14 \mathrm{MeV}$ et 3,5 MeV). Le rapport 10 entre la puissance produite et la puissance injectée donnera la preuve d'un bon confinement. Ce facteur d'amplification de l'énergie profitera de l'apport des noyaux d'hélium de 3,5 MeV, produits par la fusion et piégés dans la configuration magnétique. Ces particules chargées déposent rapidement leur énergie dans le plasma et la puissance associée s'ajoute ainsi à la puissance des systèmes de chauffage auxiliaire. L'accumulation de l'hélium, cendre de la combustion, entraînerait une dilution néfaste du combustible et le divertor devra donc assurer aussi son évacuation et sa séparation du combustible.

La valeur nominale de la puissance produite a été fixée à $500 \mathrm{MW}$ dont $80 \%$ sont portés par les neutrons de $14 \mathrm{MeV}$. Pendant ces expériences, les parois faisant face au plasma recevront un flux moyen de neutrons atteignant $0,57 \mathrm{MW} / \mathrm{m}^{2}$. La protection de l'enceinte à vide sera assurée grâce à un écran modulaire de $45 \mathrm{~cm}$ d'épaisseur disposé entre le plasma et sa paroi interne. Les neutrons seront ralentis et absorbés par les structures en acier et par l'eau $d^{\prime}$ un circuit de refroidissement qui évacuera la chaleur déposée. L'irradiation totale, pendant la vie de la machine, ne provoquera qu'une activation réduite et ne sera pas suffisante pour altérer les propriétés mécaniques. La contrainte la plus sévère porte sur le flux de neutrons s'échappant des écrans de protection et 
interagissant avec les supraconducteurs. La puissance de chauffage correspondante ne devra pas dépasser $15 \mathrm{~kW}$ alors que, pour l'ensemble de l'installation, le système de refroidissement devra évacuer $750 \mathrm{MW}$ pendant une décharge nominale.

Ces expériences apporteront des données importantes sur le comportement d'un Tokamak dans les conditions d'un réacteur, puisqu'aux études sur le plasma lui-même viendront s'ajouter le contrôle par le chauffage auxiliaire, l'alimentation en combustible et l'évacuation des cendres. Les expérimentateurs apprendront à maîtriser un plasma dont le chauffage ne dépend plus de sources auxiliaires que pour une part minoritaire. Il est prévu d'explorer des conditions où cette contribution du chauffage extérieur pourrait encore diminuer et même s'annuler, ce qui permettrait d'entrer dans le domaine de l'ignition. Mais la première investigation portera sur la validité des lois empiriques concernant le transport de l'énergie. Elles ont permis les prévisions des performances pour Iter; leur vérification dans un large domaine supplémentaire servira de base au développement à venir de la fusion par confinement magnétique.

\section{Objectif avancé}

Un second objectif vient compléter et élargir le domaine des investigations : « Le dispositif devra chercher à démontrer la possibilité d'un fonctionnement en régime permanent en utilisant une génération non inductive du courant, tout en maintenant un rapport entre puissance produite et puissance injectée au-dessus de cinq. "

Ce second volet du programme de recherche a un caractère plus exploratoire. Il s'appuie sur la possibilité de générer le courant en injectant des ondes ou des particules qui transfèrent de la quantité de mouvement aux électrons. En substituant progressivement cette méthode à l'induction, la durée des impulsions s'allongera et Iter se rapprochera d'une qualification comme configuration possible d'un réacteur. Pour cela, il faudra démontrer qu'un fonctionnement en régime permanent $n^{\prime}$ implique pas un dépôt d'énergie qui dégrade trop le facteur $\mathrm{d}^{\prime}$ amplification. On trouvera une aide précieuse dans le courant de bootstrap, qui accompagne l'existence d'un gradient de pression dans les régimes faiblement collisionnels. Mais pour que ce courant corresponde à une fraction importante du courant total sans dégrader le confinement, il faut sortir des domaines de fonctionnement habituels des Tokamaks où les nombreux résultats permettent $d^{\prime}$ 'assurer des extrapolations fiables. Des solutions innovantes sont attendues depuis la découverte de régimes à confinement améliorés, obtenus en contrôlant finement le profil de courant du plasma. Mais les incertitudes inhérentes à la recherche de ces performances ont conduit à réduire les ambitions en ce qui 
concerne le taux d'amplification de l'énergie dont la valeur visée ne devrait pas néanmoins descendre au-dessous de cinq.

\section{4 | Objectif technologique}

Le tritium brûlé dans Iter proviendra de stocks existants. Une décharge de 400 secondes consommera environ 0,4 gramme de tritium pour produire 2 GJ. Mais l'extraction, la purification et la réinjection du gaz conduiront à une circulation de 100 grammes de tritium pendant la même période. Pour les impulsions nominales de 400 secondes, le retraitement du mélange deutériumtritium-hélium en ligne n'est pas nécessaire mais, en régime permanent (audelà de 2000 secondes), la séparation des isotopes et leur réinjection auront lieu en ligne. Le cycle du tritium d'un réacteur sera ainsi étudié en grandeur réelle, à l'exception de sa génération. Toutefois, des modules tritigènes représentatifs seront inclus dans la couverture. Ils subiront une irradiation neutronique $\mathrm{d}^{\prime}$ au moins $0,5 \mathrm{MW} / \mathrm{m}^{2}$ et une irradiation cumulée totale supérieure à $0,3 \mathrm{MWa} / \mathrm{m}^{2} 1$, permettant ainsi une première validation des techniques envisagées. Après dix années d'expérimentation en vue d'atteindre les deux objectifs définis plus haut, la génération du tritium pourrait devenir nécessaire, suivant l'état des stocks disponibles, les quantités de neutrons produits et les résultats obtenus sur les modules test de la phase précédente.

Les neutrons de $14 \mathrm{MeV}$ ont une action destructrice sur les matériaux structuraux d'Iter en provoquant des déplacements d'atomes et en générant in situ de l'hydrogène et de l'hélium. L'hydrogène diffuse dans le réseau mais l'hélium $s^{\prime}$ accumule en petites poches (on dit qu'il précipite) qui altèrent les propriétés physiques du matériau, en particulier sa soudabilité. Pour protéger la structure de la chambre à vide, sa paroi interne est revêtue de modules de couverture dont l'épaisseur de $45 \mathrm{~cm}$ a été calculée pour que la dose accumulée sur les 20 années de fonctionnements ne compromette jamais sa soudabilité, nécessaire en cas réparation importante. Ces conditions d'irradiation neutronique diffèrent considérablement de celle d'un réacteur et ne constituent pas un test significatif puisque, dans Iter, l'irradiation cumulée moyenne ne dépassera pas $0,5 \mathrm{MWa} / \mathrm{m}^{2}$, alors que la première paroi $\mathrm{d}^{\prime}$ un réacteur sera soumise en permanence à une irradiation neutronique de quelques mégawatts par $\mathrm{m}^{2}$ et les considérations de rentabilité d'exploitation demandent une période de remplacement de l'ordre de trois à cinq années. Cette différence provient d'une part des flux de neutrons qui, dans Iter, seront beaucoup plus faibles que dans un réacteur et, d'autre part, du fonctionnement intermittent $d$ 'lter, sur lequel on ne

\footnotetext{
${ }^{1} 1 \mathrm{MWa} / \mathrm{m}^{2}$ correspond à une irradiation permanente pendant un an par des neutrons de
} $14 \mathrm{MeV}$ avec un flux incident en énergie de $1 \mathrm{MW} / \mathrm{m}^{2}$. 
prévoit que quelques dizaines de milliers de décharges dont la durée variera de 400 secondes à quelques milliers de secondes. L'irradiation dans Iter atteint cependant un niveau suffisant pour nécessiter impérativement la télémanipulation des éléments internes et en particuliers des modules de couverture et du divertor, qui peuvent être extraits de l'enceinte à vide et remplacés après réparation.

\section{Après Iter}

Les partenaires dans la construction d'Iter (Chine, Corée du Sud, Europe, Inde, Japon, Russie et États-Unis) ont en tête une vision sommaire, plus ou moins précise, sur le développement de la fusion après Iter et la préparation de son exploitation économique. Officiellement, un réacteur de démonstration, appelé Demo, devrait intégrer les enseignements de la machine Iter dans une installation préindustrielle couplée à des générateurs d'électricité et possédant toutes les fonctionnalités d'une centrale électrique, mais sans contrainte de rentabilité. Cette machine $n^{\prime}$ a pas encore fait l'objet d'une étude de conception détaillée. Les résultats d'Iter conditionneront le calendrier de cette étude et détermineront de nombreuses options techniques.

En revanche, depuis les débuts des recherches, les connaissances acquises ont été très rapidement appliquées à des études de réacteurs sur papier, afin de s'assurer que, malgré les contraintes scientifiques et techniques, il subsistait un créneau où un réacteur plausible pouvait trouver place. Ces études souffraient évidemment d'un manque de données dans certains domaines, en particulier pour le comportement des matériaux sous irradiation, et ces tentatives ont souvent amené des contestations. Néanmoins, l'exercice ${ }^{2}$ a été récemment tenté dans le cadre de l'European Fusion Development Agreement (EFDA), entre 1990 et 2000, avec le concours de nombreux spécialistes de l'industrie nucléaire. Les résultats donnent une idée de la distance qu'il reste à parcourir.

Ils aboutissent à quatre propositions qui diffèrent par des hypothèses de moins en moins conservatrices sur les performances de la technologie du divertor et des matériaux et sur les régimes de fonctionnement. Le réacteur le moins exigeant en progrès par rapport aux connaissances actuelles reprend simplement les hypothèses, aujourd'hui nécessairement conservatrices, utilisées pour le dessin d'Iter, mais conduit en conséquence à un schéma probablement inacceptable par de nombreux aspects. La centrale produit une puissance électrique de 1,55 GWe pour une puissance de $5 \mathrm{GW}$ dégagée par la fusion. Les principaux paramètres montrent une machine beaucoup plus volumineuse qu'lter avec $R=9,55 \mathrm{~m}$,

\footnotetext{
${ }^{2}$ Final Report of the European Fusion Power Plant Conceptual Study, April 13th 2005, EFDA-RPRE-5.0.
} 
$a=3,17 \mathrm{~m}$, élongation $=1,7, B=7$ Teslas, I = 30,5 MA dont 13,5 MA générés par bootstrap, puissance additionnelle $=246 \mathrm{MW}, 220$ millions de degrés en moyenne dans le plasma, une irradiation neutronique de $2,2 \mathrm{MW} / \mathrm{m}^{2}$ au niveau du premier mur, une charge thermique de $15 \mathrm{MW} / \mathrm{m}^{2}$ pour les zones les plus exposées du divertor. C'est la nécessité de protéger le divertor qui conduit à une taille aussi imposante, montrant ainsi l'importance de tout progrès technologique en ce domaine. L'ingénierie nucléaire s'inspire essentiellement de la technologie des réacteurs à eau pressurisée actuels. La couverture fait donc appel à un refroidissement par eau et la partie tritigène à un eutectique lithium-plomb, avec l'alliage Eurofer comme matériau de structure, en supposant qu'une optimisation lui aura permis de réunir toutes les qualités nécessaires. La conception physique ne s'écarte pas de celle d'lter et ce réacteur peut se définir comme un Iter à grande échelle. Le choix des paramètres physiques place la configuration dans une zone bien explorée où les caractéristiques du confinement sont connues. Cette assurance se paie par un courant élevé dont la génération laisse subsister des incertitudes importantes. Il reste difficile d'évaluer la faisabilité de la génération non inductive à de tels niveaux et avec un bon rendement. Iter apportera des précisions importantes à ce sujet. II en sera de même pour la stabilité, le régime de turbulence de bord et la suppression des disruptions du courant plasma qui reste un impératif.

L'étude pilotée par l'EFDA ne se limite pas à une solution unique. Conscients des problèmes posés par la taille imposante de la machine directement transposée d'Iter avec les technologies existant aujourd'hui, les experts ont également travaillé sur trois autres configurations plus audacieuses qui supposent des progrès technologiques raisonnablement accessibles (hautes températures, refroidissement à l'hélium) et des performances du plasma plus avancées qui, sans être généralement réalisées, ont déjà été obtenues expérimentalement (courants plus faibles, pressions plus fortes). Elles mettent bien en évidence l'impact important des améliorations technologiques sur le dimensionnement et la rentabilité de ces centrales. Par exemple ${ }^{3}$, ces mêmes hypothèses peuvent justifier le dessin d'une centrale produisant $1 \mathrm{GW}$ de puissance électrique à partir d'une puissance thermique de $2,6 \mathrm{GW}$, obtenus dans une machine dont les paramètres physiques sont soit ceux $d^{\prime}$ Iter, soit très voisins.

Malgré ces incertitudes, cette étude présente une grande utilité en mettant en évidence les domaines critiques où les progrès se traduisent par $d^{\prime}$ importantes améliorations au niveau du réacteur et de son exploitation. Aucun blocage mettant en cause la poursuite du programme n'est apparu aux différents groupes qui ont expertisés les diverses conceptions.

\footnotetext{
${ }^{3}$ How far is a fusion power reactor from an experimental reactor? R. Toschi, P. Barabaschi, D. Campbell et al., SOFT Conference, Madrid, Sept. 2000.
} 


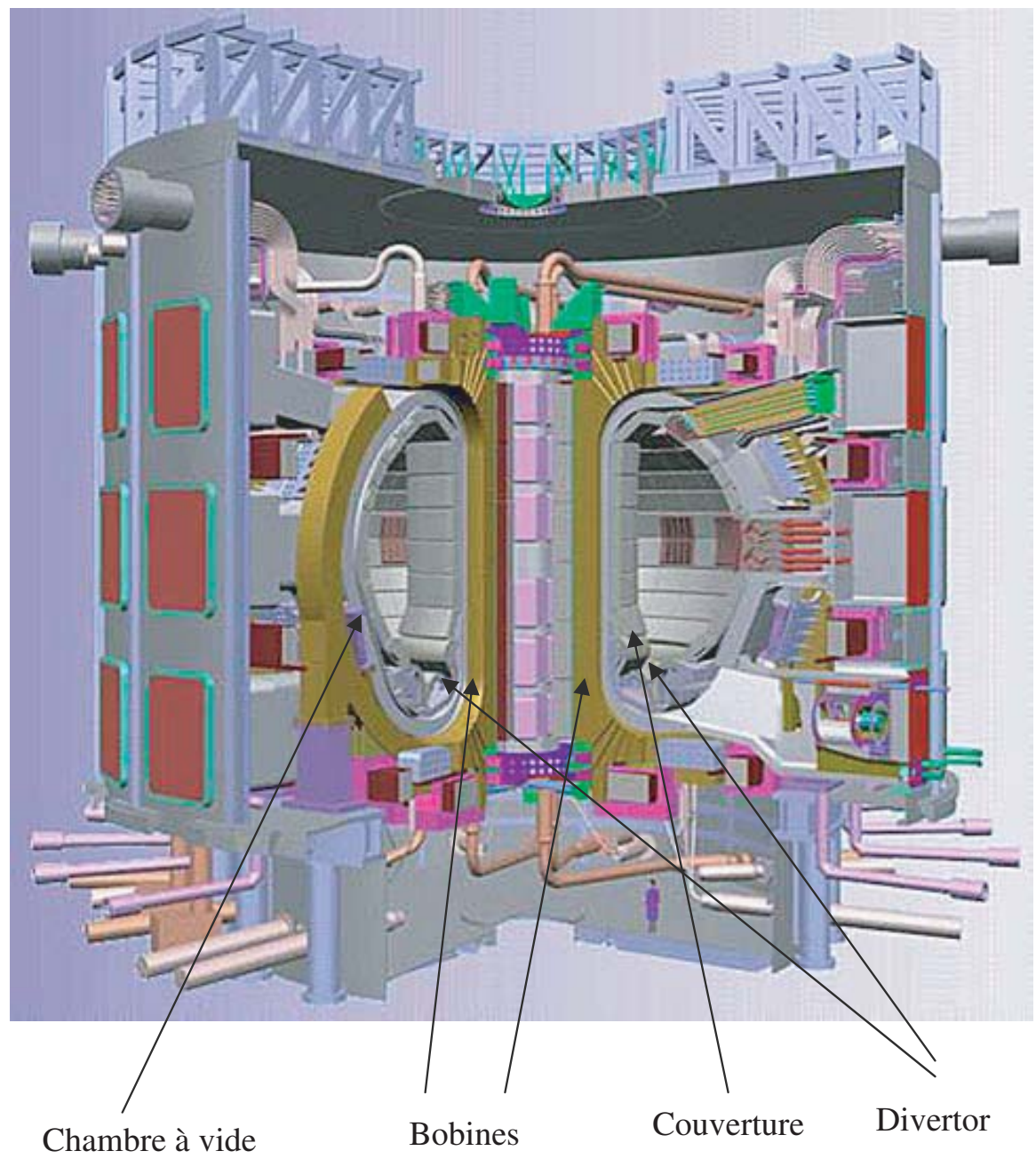

Figure 1.1

Éclaté de la machine lter. 
